\title{
Prävention
}

\section{Kann Lebensstilintervention Kosten sparen?}

Fragestellung: Wie kosteneffektiv sind Maßnahmen zur Diabetesprävention?

Hintergrund: Vielfach wird behauptet, dass Maßnahmen zur Diabetesprävention kosteneffektiv sind, ohne dass dafür eine gute Evidenzbasis existiert. Es ist davon auszugehen, dass in unterschiedlichen Gesundheitssystemen und unterschiedlichen Finanzierungsmodellen eine Kosteneffektivität unterschiedlich betrachtet werden muss. Die Diabetes Prevention Program Outcome Study (DPPOS) ergab, dass eine Lebensstilintervention über drei Jahre selbst nach zehn Jahren noch einen diabetespräventiven Effekt in der Interventionsgruppe hat. In der nun vorliegenden Studie wurde die Kosteneffektivität dieser Intervention untersucht.

Patienten und Methodik: Die Daten der Diabetes Prevention Program Outcome Study wurden hinsichtlich der Kosten untersucht. Über zehn Jahre wurden die direkten medizinischen Kosten der Lebensstilinterventionen mit den direkten medizinischen Kosten in der Metformingruppe und der Kontrollgruppe verglichen.

Ergebnisse: Die Untersuchung ergab, dass die direkten medizinischen Kosten für die Lebensstilintervention
4601 US-Dollar pro Person betrugen, verglichen mit Kosten von 2300 US-Dollar in der Metformingruppe und von 769 US-Dollar in der Placebogruppe. Die kumulierten direkten medizinischen Kosten außerhalb der Studienintervention waren in der Lebensstilgruppe 24563 US-Dollar verglichen mit 25616 US-Dollar in der Metformingruppe und 27468 US-Dollar in der Placebogruppe. Damit waren die kombinierten direkten medizinischen Kosten am höchsten in der Lebensstilinterventionsgruppe (29 164 US-Dollar) und am niedrigsten in der Metformingruppe (27915 US-Dollar). In der Placebogruppe betrugen sie 28263 US-Dollar.

Die kumulativen qualitätsadjustierten Lebensjahre (QUALY) waren am höchsten in der Lebensstilgruppe mit 6,81 Jahren gefolgt von der Metformingruppe mit 6,69 Jahren und der Placebogruppe mit 6,67 Jahren.

Wenn die aufwendige Intervention gestoppt wurde und insgesamt zu einem Preis von 3\% der Ursprungsinvestition durchgeführt wurde, ergab sich ein Kostenaufwand von 10037 US-Dollar per QUALY, was hoch kosteneffektiv ist.

Schlussfolgerung: Eine Kernintervention von drei Jahren zeigt selbst nach zehn Jahren noch eine Kosteneffektivität von Maßnahmen zur Diabetesprävention.
Kommentar: Auch wenn die Daten etwas verwirrend erscheinen und man bei den Einzelbeträgen keine großen Unterschiede finden mag, ist es trotz alledem eine Landmark-Studie die zeigt, dass eine Lebensstilintervention verglichen mit Placebo kosteneffektiv ist. Interessanterweise war die Metformin-Intervention gegenüber Placebo nur grenzwertig kostensparend. Die Autoren schreiben daher, dass beide Interventionen einen guten Gegenwert für das investierte Geld aus Sicht einer Krankenkasse ergeben.

Was bedeutet das für uns? Wir haben erstmal eine gute Basis einer über zehn Jahre dauernden Verlaufsstudie, um mit den Kostenträgern über die Kosteneffektivität von Präventionsmaßnahmen zu diskutieren. Ein Kritikpunkt hierbei kann sicherlich sein, dass die direkten medizinischen Kosten in den USA andere sind als das in Deutschland der Fall wäre und es wichtig ist, diese
Kosten auch für europäische Staaten und Deutschland zu modellieren, was derzeit gerade bearbeitet wird. Allerdings ist es so, dass die medizinischen Kosten in den USA deutlich höher sind als in Deutschland und von daher dieses Ergebnis beachtenswert ist. Die Lebensstilintervention im Rahmen der hier vorgestellten Studie hat ein Vielfaches dessen gekostet, was entsprechende Lebensstilinterventionen in Deutschland nach $\S 20$ SGB5 kosten, sodass wir davon ausgehen können, dass ein ähnliches Setting in Deutschland vielleicht sogar einen Kostenspareffekt der Lebensstilintervention zeigen würde.

\section{Prof. Dr. med. habil. Peter Schwarz}

Universität Dresden

Fetscherstr. 74

01307 Dresden

peter.schwarz@uniklinikum-dresden.de
The Diabetes Prevention Program Research Group. The 10-Year CostEffectiveness of Lifestyle Intervention or Metformin for Diabetes Prevention: An intent-to-treat analysis of the DPP/ DPPOS. Diabetes Care 2012;35(4):72330.

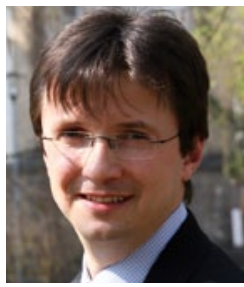

Prof. Dr. med. habil. Peter Schwarz 